\title{
Does a high-fat diet-induced obesity model brown adipose tissue thermogenesis? A systematic review
}

\author{
Gabriela S. Perez, Gabriele D.S. Cordeiro, Lucimeire S. Santos, Djane D.A. Espírito-Santo,
} Gilson T. Boaventura, Jairza M. Barreto-Medeiros

Graduate Program of Food Nutrition and Health, Department of Nutrition Science, Federal University of Bahia, Brazil

Submitted: 13 April 2018; Accepted: 20 May 2018;

Online publication: 22 July 2019

Arch Med Sci 2021; 17 (3): 596-602

DOI: https://doi.org/10.5114/aoms.2019.86781

Copyright @ 2019 Termedia \& Banach

\section{Abstract}

Introduction: In this systematic review, we analysed studies that assessed the brown adipose tissue (BAT) activity in the high-fat/cafeteria diet model of obesity in rats.

Material and methods: Scopus, PubMed, Embase, and ScienceDirect databases were searched from January 2017 to November 2017. Using specific combinations of medical subject heading (MeSH) descriptors, seven papers remained after the inclusion and exclusion criteria.

Results: Most papers showed an increase in BAT thermogenesis in rodents fed high-fat/cafeteria diet. Some studies did not mention the diet composition or housing temperature, and the most of them investigated the thermogenesis superficially, being limited to the analysis of the UCP 1 expression. Conclusions: Despite the consolidated use of high-fat/cafeteria diets as a model to induce obesity, the identification of the energy expenditure arm has been slow, especially the direct quantitative assessment of the contribution of BAT to the increase in metabolic rate in rats fed a cafeteria/high-fat diet.

Key words: rats, high-fat diet, brown adipose tissue, thermogenesis, cafeteria diet.

\section{Introduction}

The global prevalence of overweight and obesity continues to rise worldwide [1]. This situation has made studies to search for novel preventions and/or treatments an urgent matter. Although the role of fat in human obesity remains contested, models of diet-induced obesity have been preferentially used.

One of the first studies using the high-fat diet to induce obesity by nutritional intervention was published in 1959 [2]. Subsequent studies have shown that high-fat diets promote an increase in body weight, white adipose tissue, and insulin resistance, among others [3, 4], becoming one of the most widely used models to mimic human obesity.

Because recent evidence has shown that the adult human body also contains functionally distinct brown adipose tissue (BAT), this organ has garnered attention as a therapeutic intervention for obesity and metabolic diseases [5]. Due to its main function of oxidising fuels and dissipating energy in the form of heat, BAT has been recognised as an important and natural anti-obesity tool $[5,6]$.

\author{
Corresponding author: \\ Gabriela S. Perez \\ Federal University of Bahia \\ Rua Basilio da Gama \\ Campus Canela \\ 40110907, Salvador, Brazil \\ Phone: +5571982505454 \\ E-mail: perezgds@gmail.com
}


Therefore, the renewed interest in BAT thermogenesis as a key component of energy balance to reduce obesity intensifies the debate about whether BAT thermogenesis burns off excess calories in a state of positive energy balance to maintain energy homeostasis, neglecting important changes that may interfere in the results of these studies. Thus, the aim of this review is to investigate the influence of the high-fat diet (HFD)-induced obesity model in brown adipose tissue thermogenesis.

\section{Material and methods}

This systematic review was conducted from January 2017 to November 2017, using four databases: Scopus, PubMed, ScienceDirect, and Embase. The following combination of medical subject heading (MeSH) descriptors was used: high-fat diet OR cafeteria diet AND Thermogenesis OR Brown Adipose Tissue OR Bege adipose tissue OR Browning.

The first phase of the search and selection of articles were carried out by reading of the title and abstract. Articles in accordance with the eligibility criteria were included in the next screening phase. In the second phase, the selected papers were read in full, and those that fully met the criteria established in this review were included.

\section{Eligibility criteria}

For this review, only experimental studies using animals and with full text papers published in the last 5 years (2012-2017) were included. Studies that included more than one type of intervention (e.g. housing temperature, physical exercise, etc.) were included if they owned an experimental group only in the use of HFD. Papers were excluded if they used diets rich in good quality fat (e.g. polyunsaturated) or used another type of diet. Also, the non-original reviews and the clinical studies with humans or non-mammalian animal articles, or those studies that investigated the outcomes in maternal or neonatal period, were excluded.

\section{Outcome measures (dependent variables)}

Diet-induced thermogenesis measured by expression of genes related to thermogenesis. Another outcome of interest was the browning of white adipose tissue measured by gene expression or histology analysis.

\section{Systematic review process}

Titles and abstracts were assessed for full text retrieval (Perez G.S.). Full text articles were assessed against the inclusion and exclusion criteria by two independent reviewers (Perez G.S. and Cordeiro G.D.S.). To help organise the different phases of this research, we used the PRISMA flow diagram (Figure 1) [7].

\section{Results}

Seven studies [8-14] were selected after all the eligibility criteria were met (Table I). Regarding the location in which the studies were carried out, two were from Japan, and the others were from Spain, the United States of America, Sweden, Germany, and Canada. Four of these studies used the $\mathrm{C} 57 \mathrm{BL} / 6 \mathrm{~J}$ mice as an animal model for diet-induced obesity, and the others used NMRI strain mice, Wistar rats, or Sprague-Dawley.

One important measure for studies that investigate BAT activity, the housing temperature, was not mentioned in two of the papers. The others used $22-23^{\circ} \mathrm{C}$, and one also checked the diet-induced thermogenesis in rats in the thermoneutral zone $\left(30^{\circ} \mathrm{C}\right)$ and at $4^{\circ} \mathrm{C}$. Only one study was carried out using female mice, while six used male animals.

Regarding the samples, most of the articles (four) did not mentioned the number of animals per group. The remaining used around eight animals per experimental group. The diet composition was very different between all the studies. The proportion of calories from fat was $20 \%$ and $63 \%$ with HFD diet and cafeteria diet, respectively. Another important variable in the diet composition was the amount of fat in control diets, at around $10 \%$. However, two studies $[11,12]$ did not clarify the composition of the control diet, and only three

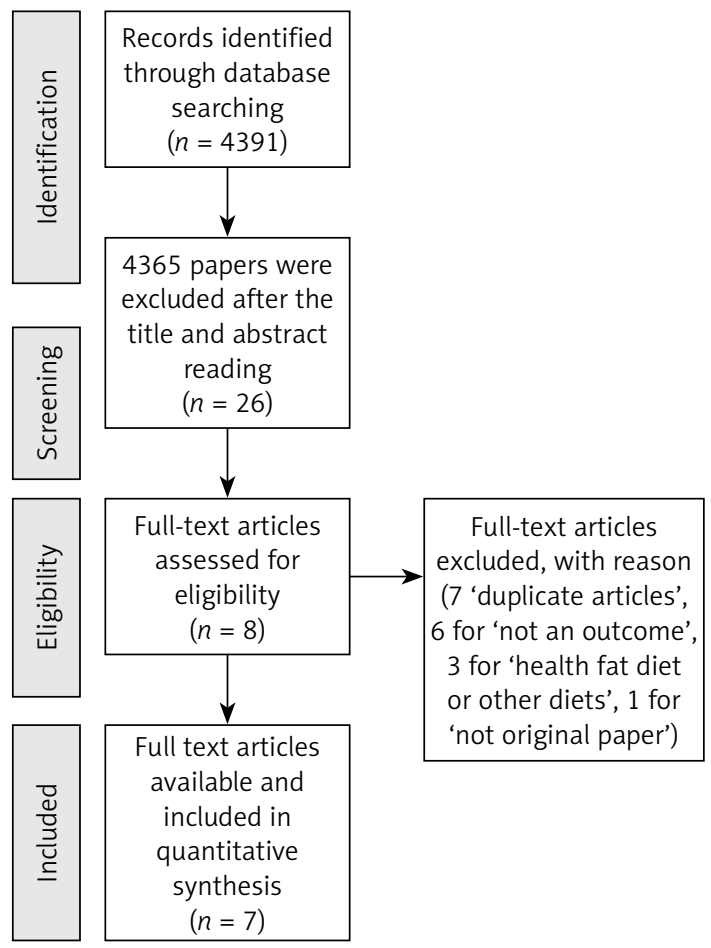

Figure 1. Adapted PRISMA flow diagram [7] systematic search and review process 


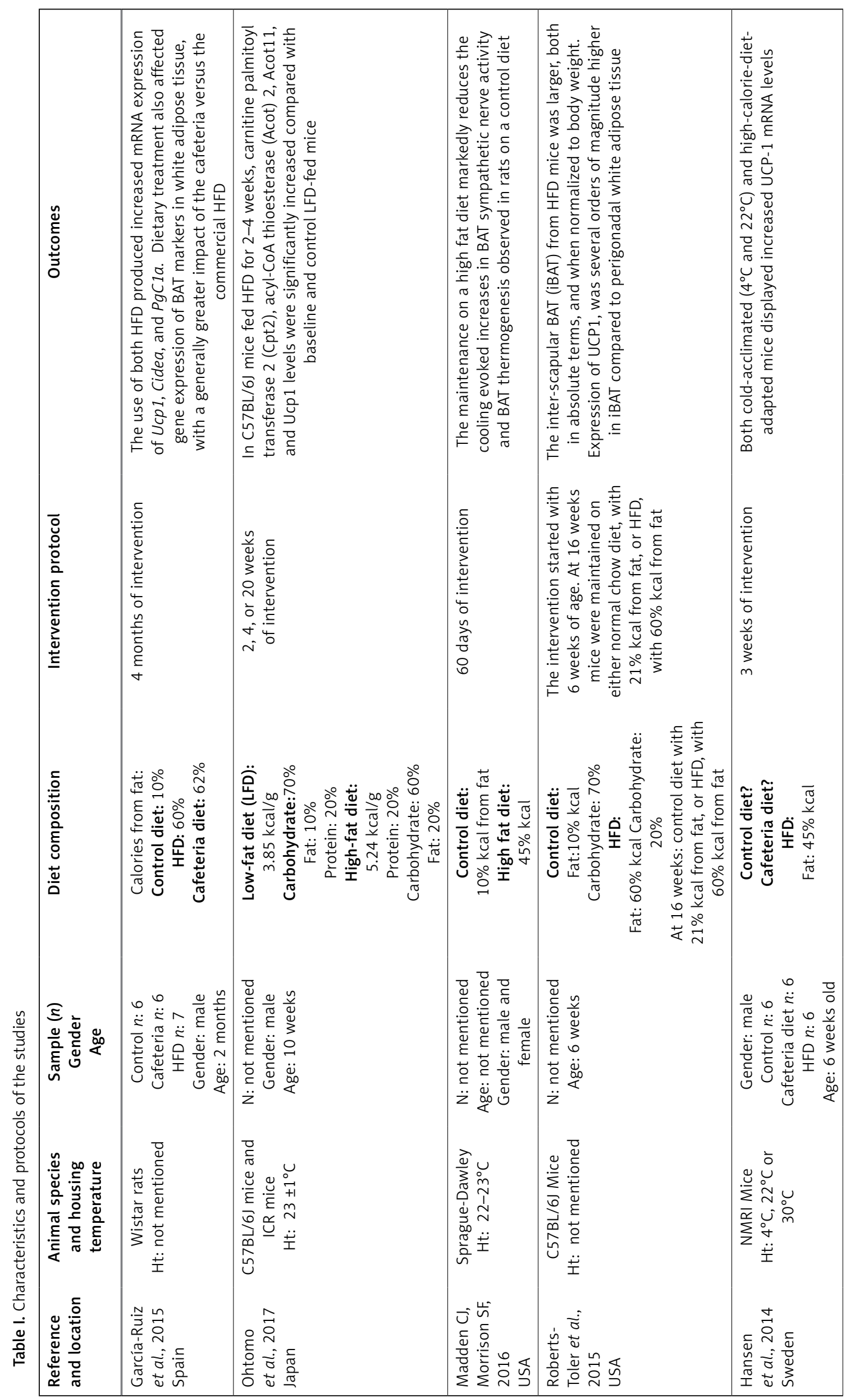




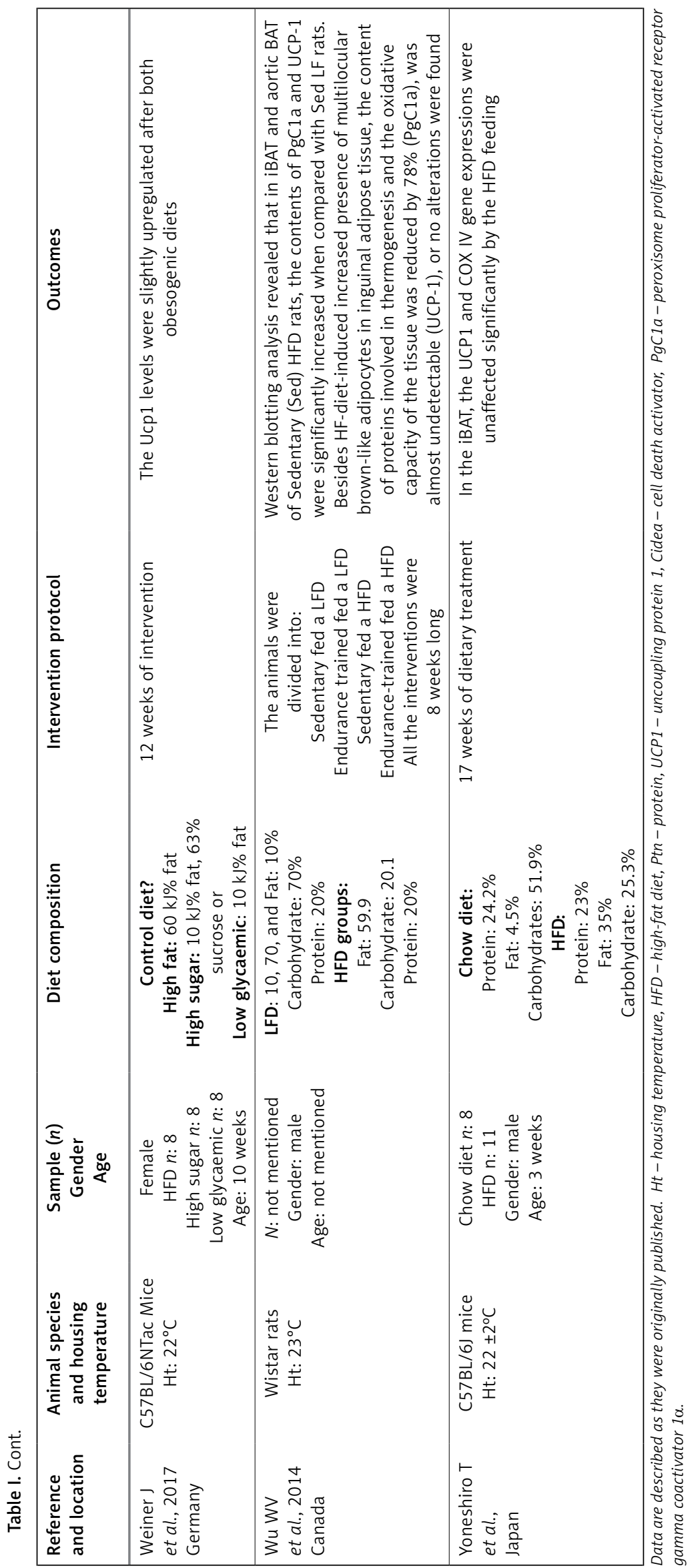


$[9,13,15]$ informed about the total composition of carbohydrate, protein, and fat of the diet.

Regarding the intervention protocol, the animals were exposed to HFD or cafeteria diet for 2 weeks [9], 3 weeks [11], 4 weeks [9], 8 weeks [13, 16], 10 weeks [10], 12 weeks [12], 16 weeks [8], 17 weeks [15], and 20 weeks [9]. Despite all the methodological differences, five studies verified increased brown adipose tissue activity in animals that consumed a HFD or cafeteria diet, and this measure was basically verified through the increased messenger ribonucleic acid (mRNA) expression of classical brown adipocyte genes in BAT, specially the uncoupling protein 1 (UCP-1).

\section{Discussion}

The animal models of obesity, obtained by the introduction of a high-fat diet, are widely used because this template reflects similar metabolic changes to those found in human obesity [17] A better understanding if this kind of diet can stimulate thermogenesis and is crucial to affirm its real role in the genesis of obesity. Thus, this review investigated the influence of a HFD-induced obesity model in brown adipose tissue thermogenesis.

Observing the location of the studies, there is a predominance of experiments carried out on the European continent. However, the growing interest in the thermogenic properties of brown adipose tissue is noticeable in all parts of the world.

The animal species most used in the studies were $\mathrm{C} 57 \mathrm{BL} / 6 \mathrm{~J}$ mice, a genetic model for variation in diet-induced obesity [18]. Because under-reporting is an important bias in epidemiological studies on diet and obesity in human subjects, animal models have been widely utilised for experiments on dietary obesity. Both rats and mice provide ideal animal models for biomedical research and comparative medicine studies because they have many similarities to humans in terms of anatomy and physiology. Rats, mice, and humans each have approximately 30,000 genes of which nearly $95 \%$ are shared by all three species [19].

In this review, we observed that three of the studies did not mention the housing temperature. This information is crucial once BAT is activated by sympathetic noradrenergic stimuli and cold is the main physiological stimuli to this noradrenergic activation [5]. Despite the fact that most of the facilities for rats and mice are operated at a temperature of $20-24^{\circ} \mathrm{C}$ [20], the rodent thermoneutral zone is between $26^{\circ} \mathrm{C}$ to $34^{\circ} \mathrm{C}$ [21]. It means that the temperatures usually used to house the rodents may stimulate BAT thermogenesis. Because the ambient temperature in which rats are maintained is normally below their thermoneu- tral zone, their cooling-induced activation of BAT makes an important contribution to their overall energy expenditure.

One of the pioneering studies on diet-induced thermogenesis [22] observed that cafeteria-diet-fed rats in $29^{\circ} \mathrm{C}$ housing temperature did not show an increase in BAT activity, whereas those exposed to a temperature of $24^{\circ} \mathrm{C}$ had an increase in UCP-1 expression. One of the eligible papers found in this systematic review [11] observed that both cold-acclimatised $\left(4^{\circ} \mathrm{C}\right.$ and $\left.22^{\circ} \mathrm{C}\right)$ and high-calorie-diet-adapted mice displayed increased UCP-1 mRNA levels, pointing out a possible inadequacy in the housing temperature used in experiments with rodents, especially those aimed at inducing obesity.

Madden [23] also checked the effect of HFD on the sympathetic activation of BAT through cold defensive activation of BAT in rats. They observed that maintenance on HFD markedly reduces the cooling-evoked increases in BAT sympathetic nerve activity and BAT thermogenesis in rats. This reduction in BAT activity is dependent on the glutamatergic excitation of nucleus of the tractus solitarii neurons by a population of vagal afferents. Thus, consumption of HFD enhances a vagal afferent mechanism that chronically reduces BAT energy expenditure and thereby contributes to high-fat obesity. Therefore, it is important not only to adjust the temperature currently used in research laboratories, but also to mention this in the articles, especially those that aim to observe BAT thermogenesis.

Most of the selected articles in this review used male animals, and one also used females. Probably this preference may be related to the existing biological structure between the sexes It has been justified due to the variable nature of female data caused by hormonal fluctuations associated with the female's reproductive cycle, in spite of a lack of data in support of this position [24]. The amount of hormone is related to metabolic alterations such as leptin and adiponectin, and it is higher in females, thus influencing the food and energy intake, as well as weight gain interference. A review paper published in 2010 showed that male mice and rats are introduced as 'gold standards' for studying dietary obesity [25]. This might be because of the oestrous cycle of the female animals, which is repeated every 4-6 days and can affect the food intake of the animal during this period [26].

In fact, Roca et al. [27] observed that, although cafeteria-fed female rats had an increase in BAT mass, which may be related an increase of cell size due to enhanced lipid deposition, there was no increase of the thermogenic potential per gram of tissue. Similar results were found by Oliver 
et al. [28], where the high-fat fed females had no increase in UCP-1 expression in BAT despite the overweight and high levels of serum leptin. Failure to activate BAT could be the reason for the higher body weight gain of female rats compared with males. However, both papers found in this review had increased BAT activity after HFD feeding, even in female animals.

Five of the selected papers showed increased BAT thermogenesis despite all methodological differences. The main parameter measured to determine the activity of BAT was the expression of UCP-1, a protein that facilitates proton re-entry into the mitochondrial matrix, circumventing adenosine triphosphate (ATP) synthase. Specific UCP-1 amounts per milligram of BAT tissue or per milligram of total protein might reflect absolute changes in total body UCP-1 mass quite well [29]. Other proteins are also expressed abundantly in mouse BAT and were investigated in this review's papers as Cidea, a member of a novel family of proapoptotic proteins, and $\mathrm{PgC} 1 \mathrm{a}$, the master regulator of UCP1-mediated thermogenesis in BAT [30]. Because BAT is densely packed with mitochondria and requires fatty acid oxidation to fuel heat generation, and the mitochondrial long chain fatty acid $\beta$-oxidation requires successive carnitine acyltransferases to translocate acyl-CoAs from the cytoplasm into the mitochondrial matrix, one study also analysed the gene expression of Cpt2, Acyl CoA, and Acot11 [9].

The analysed studies demonstrated the effect of HFD in promoting adaptive thermogenesis in rodents, regardless of diet composition. Although all studies used HFD, the percentage of this nutrient varied considerably, as well the other macronutrients. The diet composition can directly influence the energy balance, being the diet-induced thermogenesis dependent on the distribution of carbohydrates, proteins and lipids, reaching $5-15 \%$ of the energy expenditure [31].

Usually HFD within the range of $30-78 \%$ of total energy intake are used for studying obesity in rats and mice [32] by adding a particular fat to the animal's diet or using a variety of highly palatable, energy-dense foods that are prevalent in Western society (cafeteria diet). In this review, the proportion of calories from fat found was among $20 \%$ and $63 \%$. Even with only $20 \%$ of the caloric contribution from fat, there was a change in the brown adipose tissue activity probably in response to fat overload.

The characteristics of the used diets differed within and between laboratories in macronutrient composition and energy density. Two studies did not clarify the composition of control diet, and only two informed about the total composition of carbohydrate, protein, and fat of the diet. It is known that this difference in the diet composition might reflect the current food pattern, nutritionally unbalanced, rich in high-calorie content, increasing the risk for chronic diseases in adults [33, 34]. However, despite being a high-fat diet model, not only fat is used to generate heat, but also carbohydrate and protein increase the BAT thermogenic capacity [35]; therefore, this could have confounding effects arising from comparisons made with the high-fat/cafeteria diets.

Thus, even consuming HFD for short periods, it is possible to check for increased thermogenic activity of brown fat, which explains the reduction in adiposity expected from the level of energy intake. This concept should be taken into consideration when a diet-induced obesity mouse model is used in studies. While there has been significant progress in understanding how food intake is regulated, the identification of the energy expenditure arm has been slow, specially the direct quantitative assessment of the contribution of BAT to the increase in metabolic rate in rats fed a cafeteria/ high-fat diet.

Given the great and consolidated use of highfat/cafeteria diets as a model to induce obesity, as well the emergency to understanding the role of BAT as a new target to combat obesity, it is extremely urgent to verify the role of these diets in the activity of brown adipose tissue.

\section{Acknowledgments}

The authors thank Federal University of Bahia and National Council for Scientific and Technological Development (CNPq).

This study was financed in part by the Coordenação de Aperfeiçoamento de Pessoal de Nível Superior - Brasil (CAPES) - Finance Code 001.

\section{Conflict of interest}

The authors declare no conflict of interest.

\section{References}

1. Malik VS, Willett WC, Hu FB. Global obesity: trends, risk factors and policy implications. Nat Rev Endocrinol 2013; 9: 13-27.

2. Masek J, Fabry P. High-fat diet and the development of obesity in albino rats. Experientia 1959; 15: 444-5.

3. dos Santos Perez G, Santana dos Santos L, dos Santos Cordeiro $\mathrm{G}$, et al. Maternal and post-weaning exposure to a high fat diet promotes visceral obesity and hepatic steatosis in adult rats. Nutr Hosp 2015; 32: 1653-8.

4. Bastard JP, Maachi M, Lagathu C, et al. Recent advances in the relationship between obesity, inflammation, and insulin resistance. Eur Cytokine Netw 2006; 17: 4-12.

5. Cannon B, Nedergaard J. Brown adipose tissue: function and physiological significance. Physiol Rev 2004; 84: 277-359.

6. Bartelt A, Heeren J. Adipose tissue browning and metabolic health. Nat Rev Endocrinol 2014; 10: 24-36. 
7. Prisma. Prisma Flow Diagram. 2009 [cited 201721 April]; Available from: http://www.prisma-statement.org/PRISMA Statement/FlowDiagram.aspx.

8. Garcia-Ruiz E, Reynés B, Díaz-Rúa R, Ceresi E, Oliver $P$, Palou A. The intake of high-fat diets induces the acquisition of brown adipocyte gene expression features in white adipose tissue. Int J Obes (Lond) 2015; 39: 1619-29.

9. Ohtomo T, Ino K, Miyashita R, et al. Chronic high-fat feeding impairs adaptive induction of mitochondrial fatty acid combustion-associated proteins in brown adipose tissue of mice. Biochem Biophys Rep 2017; 10: 32-8.

10. Roberts-Toler C, O'Neill BT, Cypess AM. Diet-induced obesity causes insulin resistance in mouse brown adipose tissue. Obesity 2015; 23: 1765-70.

11. Hansen IR, Jansson KM, Cannon B, Nedergaard J. Contrasting effects of cold acclimation versus obesogenic diets on chemerin gene expression in brown and brite adipose tissues. Biochim Biophys Acta 2014; 1841: 1691-9.

12. Weiner J, Rohde K, Krause K, et al. Brown adipose tissue (BAT) specific vaspin expression is increased after obesogenic diets and cold exposure and linked to acute changes in DNA-methylation. Mol Metab 2017; 6: 482-93.

13. Wu MV, Bikopoulos G, Hung S, Ceddia RB. Thermogenic capacity is antagonistically regulated in classical brown and white subcutaneous fat depots by high fat diet and endurance training in rats: impact on whole-body energy expenditure. J Biol Chem 2014; 289: 34129-40.

14. Neyrinck AM, Bindels LB, Geurts L, Van Hul M, Cani PD, Delzenne NM. A polyphenolic extract from green tea leaves activates fat browning in high-fat-diet-induced obese mice. J Nutr Biochem 2017; 49: 15-21.

15. Yoneshiro T, Kaede R, Nagaya K, et al. Royal jelly ameliorates diet-induced obesity and glucose intolerance by promoting brown adipose tissue thermogenesis in mice. Obes Res Clin Pract 2018; 12 (Suppl 2): 127-37.

16. Neyrinck AM, Bindels LB, Geurts L, Van Hul M, Cani PD, Delzenne NM. A polyphenolic extract from green tea leaves activates fat browning in high fat diet-induced obese mice. J Nutr Biochem 2017; 49: 15-21.

17. Chakraborty TR, Donthireddy L, Adhikary D, Chakraborty S. Long-term high fat diet has a profound effect on body weight, hormone levels, and estrous cycle in mice. Med Sci Monit 2016; 22: 1601-8.

18. Chu DT, Malinowska E, Jura M, Kozak LP. C57BL/6J mice as a polygenic developmental model of diet-induced obesity. Physiol Rep 2017; 5: e13093.

19. Waterston RH, Lindblad-Toh K, Birney E, Rogers J et al.; Mouse Genome Sequencing Consortium. Initial sequencing and comparative analysis of the mouse genome. Nature 2002; 420: 520-62.

20. Havenaar R, Meijer JC, Morton DB, Ritskes Hoitinga J Zwart P. Biology and husbandry of laboratory animals. In: Principles of Laboratory Animal Science. van Zutphen LF, Baumans V, Beynen AC (eds.). Elsevier, Amsterdam 2001; 19-76.

21. Romanovsky AA, Ivanov Al, Shimansky YP. Selected contribution: ambient temperature for experiments in rats: a new method for determining the zone of thermal neutrality. J Appl Physiol (1985) 2002; 92: 2667-79.

22. Rothwell NJ, Stock MJ. Influence of environmental temperature on energy balance, diet-induced thermogenesis and brown fat activity in 'cafeteria'-fed rats. Br J Nutr 1986; 56: 123-9.

23. Madden CJ. Consumption of a high fat diet inhibits sympathetic outflow to brown adipose tissue (BAT) via va- gal afferent activation of neurons in the Nucleus Tractus Solitarius (NTS). Auton Neurosci 2015; 192: 13.

24. Becker JB, Prendergast BJ, Liang JW. Female rats are not more variable than male rats: a meta-analysis of neuroscience studies. Biol Sex Differ 2016; 7: 34.

25. Hariri N, Thibault L. High-fat diet-induced obesity in animal models. Nutr Res Rev 2010; 23: 270-99.

26. Andersen ME, Clewell HJ $3^{\text {rd }}$, Gearhart J, Allen BC, Barton HA. Pharmacodynamic model of the rat estrus cycle in relation to endocrine disruptors. J Toxicol Environ Health 1997; 52: 189-209.

27. Roca P, Rodriguez AM, Oliver P, et al. Brown adipose tissue response to cafeteria diet-feeding involves induction of the UCP2 gene and is impaired in female rats as compared to males. Pflugers Arch 1999; 438: 628-34.

28. Oliver P, Sánchez J, Caimari A, Miralles O, Picó C, Palou A. The intake of a high-fat diet triggers higher brown adipose tissue UCP1 levels in male rats but not in females. Genes Nutr 2007; 2: 125-6.

29. Fromme T, Klingenspor M. Uncoupling protein 1 expression and high-fat diets. Am J Physiol Regul Integr Comp Physiol 2011; 300: R1-8.

30. Sharma BK, Patil M, Satyanarayana A. Negative regulators of brown adipose tissue (BAT)-mediated thermogenesis. J Cell Physiol 2014; 229: 1901-7.

31. Kinabo JL, Durnin JV. Thermic effect of food in man: effect of meal composition, and energy content. Br J Nutr 1990; 64: 37-44.

32. Buettner R, Scholmerich J, Bollheimer LC. High-fat diets: modeling the metabolic disorders of human obesity in rodents. Obesity (Silver Spring) 2007; 15: 798-808.

33. Mazidi M, Wong ND, Katsiki N, Mikhailidis DP, Banach M. Dietary patterns, plasma vitamins and trans fatty acids are associated with peripheral artery disease. Lipids Health Dis 2017; 16: 254.

34. Mazidi M, Mikhailidis DP, Banach M. Higher dietary acid load is associated with higher likelihood of peripheral arterial disease among American adults. J Diabetes Complications 2018; 32: 565-9.

35. de Franca SA, dos Santos MP, Przygodda F, et al. A low-protein, high-carbohydrate diet stimulates thermogenesis in the brown adipose tissue of rats via ATF- 2 . Lipids 2016; 51: 303-10. 